\title{
Study on Voltage Fluctuation Tracking of Distribution System Based on HHT
}

\author{
Yanqing Li, Yan Fu, Feilong Wang* \\ School of Electrical and Electronic Engineering, North China Electric Power University, Baoding 071000, China
}

\begin{abstract}
Voltage fluctuation is an essential problem exiting in distribution network. This paper applies Hilbert Huang transform (HHT) algorithm to dealing with voltage fluctuation tracking. An adaptive method based on waveform matching is presented to prevent the endpoint effects in mode decomposition. Numerical examples are given and the simulation verifies the practicality of the proposed method.
\end{abstract}

Keywords: Hilbert Huang transform, voltage fluctuations, tracking, simulation

\section{Introduction}

In the distribution network there are serious voltage fluctuation problems, which affect people's lives and production and prevent the development of many enterprises. Distribution network has the characteristics of low load density, big difference between peak and valley load, far away from the power sources, high vacancy rates, low natural power factor, and so on, which result in frequent voltage fluctuations of the distribution network [1].

If the voltage quality of distributed network can not be guaranteed, it will threaten the stability of the power system. The commonly used method to improve the voltage quality of distribution network is the reactive power control. To achieve the voltage control of distribution network, quick detection of voltage fluctuation of the distribution network must be the first, and for this, the Hilbert Huang Transform algorithm (HHT) is presented in this paper for the distribution network voltage fluctuation tracking. In order to prevent the endpoint effects in the process of modal decomposition, the adaptive continuation method, based on waveform matching is applied in the signal processing. Finally, numerical examples are simulated by the method of MATLAB simulation, which verify the practicality of HHT in the voltage fluctuation of distributed network.

\section{Technology of Voltage Fluctuation Detection of Distributed Network}

\subsection{Introduction of $H H T$}

HHT is composed of empirical mode decomposition (EMD) and Hilbert transform [2]. With this method, we can decompose any signal into a finite number of intrinsic mode functions (IMF), and then we need to calculate the instantaneous frequency and instantaneous amplitude of each IMF according to the Hilbert transform. However, the end effect is an unavoidable phenomenon in HHT. During the process of EMD, non-stationary signals at both ends of the data will produce divergent phenomena. As the result of divergence will gradually contaminate the entire data sequence, it may lead to serious distortion [3].

First, according to the maximum points and minimum points of the signal which is labeled as $x(t)$, we

\footnotetext{
* Manuscript received June 12, 2012; revised July 15, 2012.

Corresponding author. Tel.: +86-31-27522215; E-mail address: wangfeilong111@126.com.
} 
obtain the average of the last envelop and the next envelop according to (1)

$$
\mu_{1}=\frac{1}{2}\left[x_{1}(t)+x_{2}(t)\right]
$$

where $x_{1}(t)$ and $x_{2}(t)$ respectively represents the last envelop and the next envelop, and $\mu_{1}$ represents the average of $x_{1}(t)$ and $x_{2}(t)$. Then we calculate the difference between $x(t)$ and $\mu_{1}$ according to (2). the difference between $x(t)$ and $\mu_{1}$ is labeled as $\theta_{1}$.

$$
\theta_{1}=x(t)-\mu_{1}
$$

If $\theta_{1}$ satisfies the conditions of IMF it becomes the first IMF component, otherwise we treat $\theta_{1}$ as the original signal and repeat the above process for k times until $\theta_{1 k}$ satisfies the conditions of IMF. After this we can obtain the first IMF component, which is clearly indicated by $\theta_{1 k}=\theta_{1(k-1)}-\mu_{1 k}$.We can use $S_{D}$, whose expression is as (3), to judge whether each filtered result is IMF component.

$$
S_{D}=\sum_{t=0}^{n}\left|\frac{\left|\theta_{1(k-1)}(t)-\theta_{1 k}(t)\right|^{2}}{\theta_{1(k-1)}^{2}(t)}\right|
$$

In (3), we need to get the value of $S_{D}$ according to the actual requirements. When $\theta_{1 k}$ meets the requirements of $S_{D}$, we take $\alpha_{1}$ as the first IMF component, that is to say $\alpha_{1}=\theta_{1 k}$. So we separate $\alpha_{1}$ from the original signal $x(t)$, and the remaining component can be expressed by (4).

$$
r_{1}=x(t)-\alpha_{1}
$$

Then we take $r_{1}$ as the newly obtained original signal $x(t)$ and repeat the above processes, so we can naturally obtain other components just like $\alpha_{2}, \alpha_{3}, \alpha_{4}, \cdots$. The decomposition process will continue until $r_{n}$ runs into monotonic trend or $\left|r_{n}\right|$ is very small. So the result of EMD can be described by (5).

$$
x(t)=\sum_{i=1}^{n} \alpha_{i}(t)+r_{n}
$$

Hilbert transform on the IMF component is labelled $\alpha_{i}(t)$ which is done through (6).

$$
H_{i}(t)=\frac{1}{\pi} \int_{-\infty}^{+\infty} \frac{\alpha_{i}(\tau)}{t-\tau} d \tau
$$

The inverse transform is as (7).

$$
\alpha_{i}(t)=\frac{1}{\pi} \int_{-\infty}^{+\infty} \frac{H_{i}(\tau)}{t-\tau} d \tau
$$

The obtained analytic signal $A(t)$ can be described by (8).

$$
A_{i}(t)=\alpha_{i}(t)+j H_{i}(t)=a_{i}(t) e^{j \phi_{i}(t)}
$$

Among (8), instantaneous amplitude is described as $a_{i}(t)=\sqrt{\alpha_{i}^{2}(t)+H_{i}^{2}(t)}$ and its phase is $\phi_{i}(t)=\arctan \left(H_{i}(t) / \alpha_{i}(t)\right)$.The instantaneous frequency can be expressed by (9).

$$
f_{i}(t)=\frac{1}{2 \pi} \frac{d \phi_{i}(t)}{d t}
$$




\subsection{End effect}

The principle of EMD shows the process of each IMF striking we need to calculate the local average value of the last envelope and the next envelope. Local maximum and minimum of each envelope is given by the cubic spline interpolation algorithm [4]. Because the two endpoints of signal are not necessarily maximum points or minimum points, they are unable to provide the conditions required for spline interpolation. This will lead to divergence phenomenon, making the decomposition results in serious distortion [4].

\subsection{Adaptive wave matching technology for signal}

In the suppression of the end effect, the commonly used methods include mirror extension method, neural network extension method, continuation method based on polynomial fitting and so on. All these methods can suppress the end effect, but there still exist some problems [5]

For effective monitoring of the distribution network voltage fluctuations, adaptive waveform matching method is applied to solve the end effect. The main idea of the waveform matching method is that according to the law of nature all signals always change according to certain rules, so it is assumed that the development trend of waveform at the boundary will be reflected by that of the internal signal. Particularly for signals with strong regularity, this feature will become more apparent. In order to test the truth extent of the extended waveform, the concept of waveform matching degree is introduced in this paper. Assuming that $f_{1}(t)$ and $f_{2}(t)$ are two sets of data sequences, and the length of the sets is $N$. $S_{1}\left(t, f_{1}(t)\right)$ and $S_{2}\left(t, f_{2}(t)\right)$ are respectively two points on $f_{1}(t)$ and $f_{2}(t)$, so the waveform matching degree of $f_{1}(t)$ and $f_{2}(t)$ opposed to $S_{1}\left(t, f_{1}(t)\right)$ and $S_{2}\left(t, f_{2}(t)\right)$ can be obtained by the following steps.

- Pan $f_{1}(t)$ in order to achieve the coincidence of $S_{1}$ and $S_{2}$,so $f_{1}(t)$ is replaced by the newly obtained $f_{1}^{\prime}(t)$.

- The waveform matching degree of $f_{1}(t)$ and $f_{2}(t)$ opposed to $S_{1}\left(t, f_{1}(t)\right)$ and $S_{2}\left(t, f_{2}(t)\right)$ can be described by (10).

$$
m d\left(f_{1}(t), f_{2}(t), \quad S\right)=\sum_{j=1}^{N}\left(f_{2}(j)-f_{1}^{\prime}(j)\right)^{2}
$$

The detailed steps of waveform matching method are as follows.

- Obtain all the extreme points $M_{i}$ of the original signal $f_{1}(t)$ and classify all the maximum points into a collection named $\left\{M_{i, \max }\right\}$ and the minimum points $\left\{M_{i, \min }\right\}$.

- Assuming that $M_{0}$ is the first maximum point and $M_{1}$ is the first minimum point, and the waveform from the starting point to $M_{1}$ is labelled as $s d_{0}$, whose length is $l$.

- FirsObtain the waveform matching degree $m d_{i}$ of all the $M_{i, \max }$ from the maximum points collection opposed to $s d_{0}$.

- Choose the band $s d_{i}$, whose waveform matching degree $m d_{i}$ is the least, and if $m d_{i}<\alpha \cdot l$ ( $\alpha$ is a constant, whose value is determined according to the requirements of the matching accuracy), $m d_{i}$ is taken as the extended waveform on the left side, otherwise goes to step (5).

- Take the mean of the two adjacent maximum points of the original signal as the extended maximum point, and the mean of the two minimum points as the extended minimum point.

\section{Simulation and Analysis}

Assuming that the voltage of a distribution network fluctuates under disturbance, the mathematical 
expression is given in (11) and the waveform is shown in Fig. 1.

$$
u(t)=\left\{\begin{array}{lc}
\cos (100 \pi t), & \text { else } \\
0.7 \cos (100 \pi t), & 0.1<t<0.2
\end{array}\right.
$$

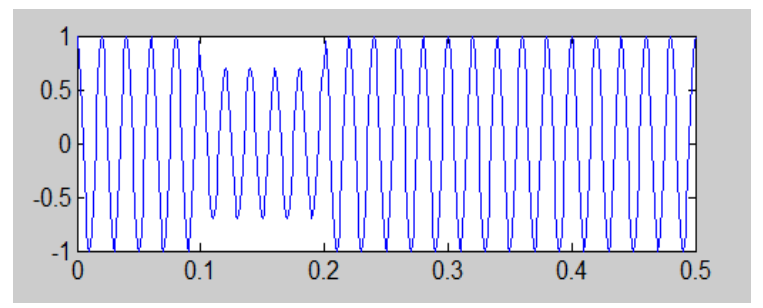

Fig. 1. Voltage waveform 1
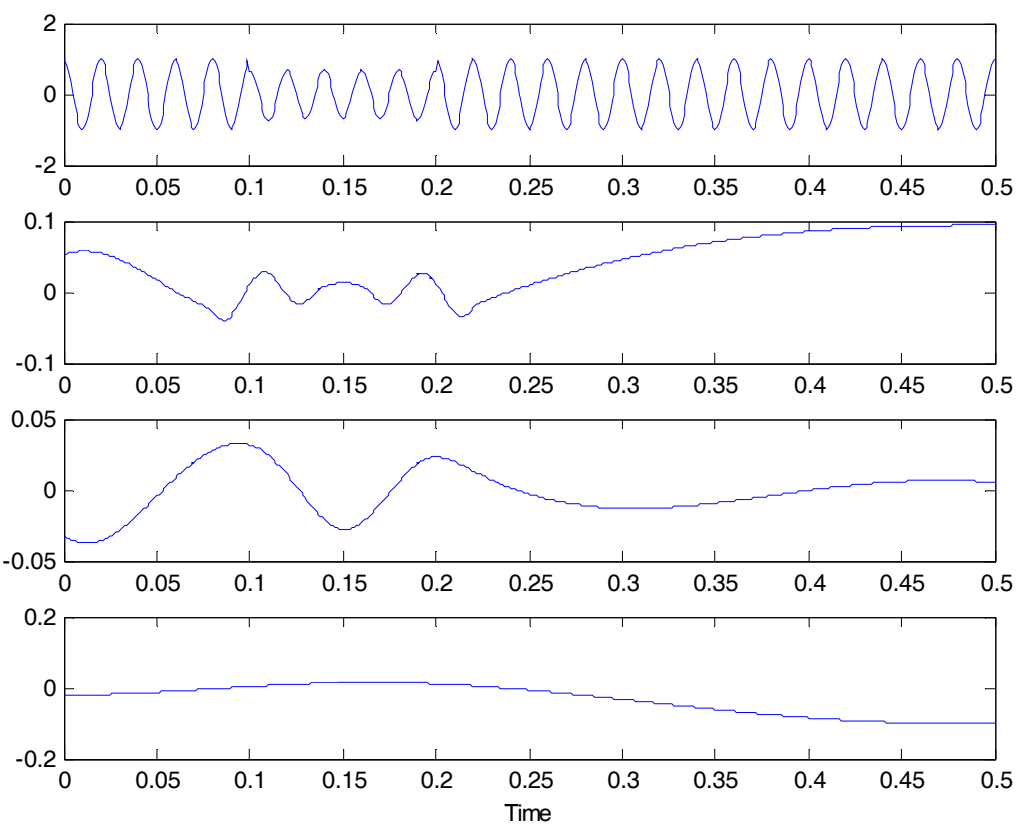

Fig.2. Results of EMD decomposition.
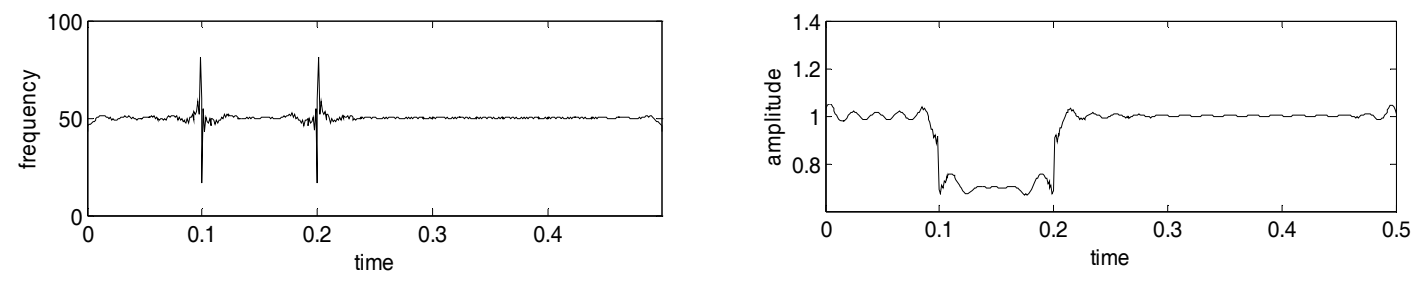

Fig.3. Amplitude-time, frequency-time response curve

The EMD decomposition results and the amplitude-time, frequency-time response curve of IMF1 simulated by Matlab are showed in Fig. 2 and Fig. 3.

In this example, the voltage of distribution network decreases to 0.7 times of the rated value under disturbance during the period of 0.1-0.2 second. From the amplitude-time response curve of IMF1 we can easily find the voltage during the period of 0.1-0.2 second is about 0.7 times that of the rated value and from the frequency-time response curve we can see that at the moment of 0.1 second and 0.2 second the frequency has a serious deviation from its rated value $(50 \mathrm{~Hz})$. The highest frequency is up to $72.3 \mathrm{~Hz}$ while the lowest is only $21.4 \mathrm{~Hz}$. The entire phenomena prove that the voltage does fluctuate at the moment of 0.1 second and 0.2 second.

Assuming that the voltage of distribution network is disturbed by instantaneous interference at the 
moment of 0.2 second, that is to say voltage runs into mutation at the moment of 0.2 second. The waveform of this is shown in Fig. 4.

The EMD decomposition results and the amplitude-time, frequency-time response curve of IMF1 simulated by Matlab are showed in Fig. 5 and Fig. 6.

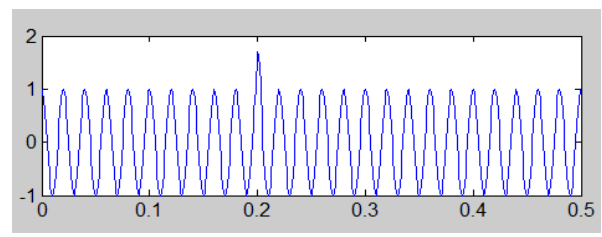

Fig. 4. Voltage waveform 2
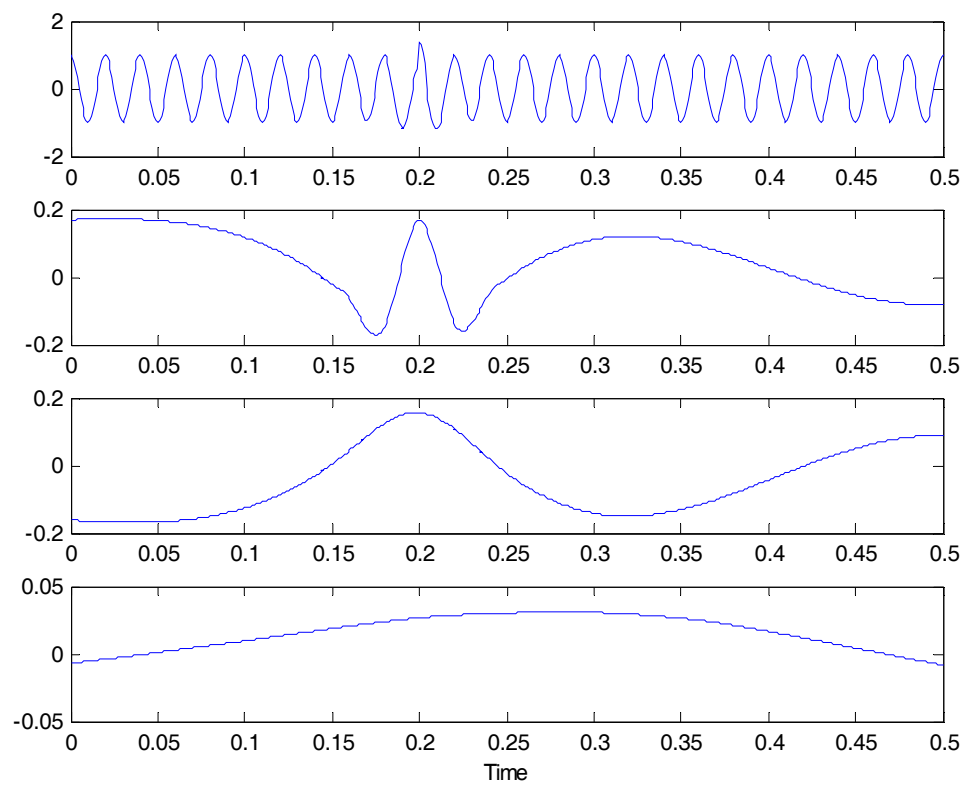

Fig. 5. Results of EMD decomposition.
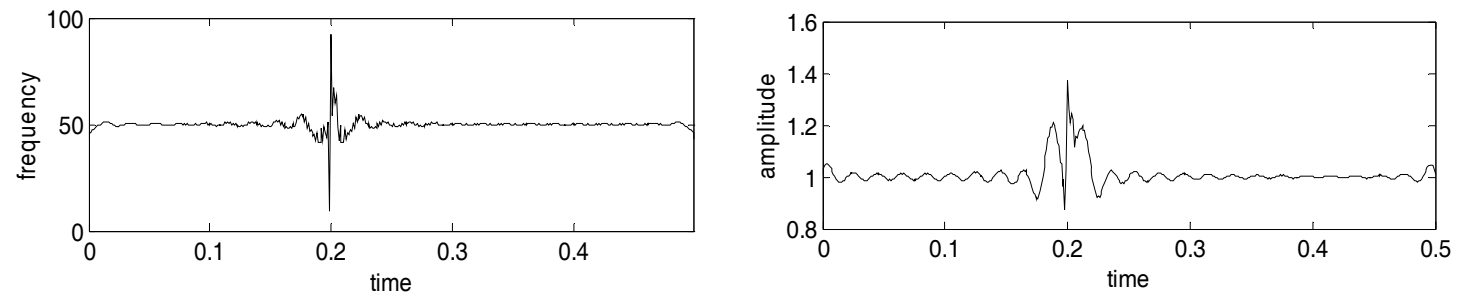

Fig. 6. Amplitude-time, frequency-time response curve.

In the example, the voltage of distribution network increases to about 1.3 times of the rated value under disturbance at the moment of 0.2 second. From the amplitude-time response curve of IMF1 we can easily find voltage at 0.2 second fluctuates and the instantaneous frequency at the moment of 0.2 second has a serious deviation from its rated value $(50 \mathrm{~Hz})$. The highest frequency is up to $93.6 \mathrm{~Hz}$ while the lowest is only $7.9 \mathrm{~Hz}$. The entire phenomena prove that voltage does fluctuate at the moment of 0.2 second.

\section{Conclusion}

HHT is applied to achieve the voltage fluctuation tracking of the distributed network in this paper and in order to solve the end effect during the process of EMD, the waveform matching method is used to extend the waveform. The simulation results show that HHT can effectively track the voltage fluctuations 
of distributed network and the exact moment of the fluctuation can be accurately obtained. What's more, the disturbance intensity can be qualitatively understood by the amplitude of IMF1.Further results on EMD's eigenvalue extraction, and a detailed analysis can quantify the disturbance voltage parameters. The simulation results in this paper provide theoretical guidance to the voltage fluctuation of distributed network, but the distribution network is very complicated, the algorithm should be improved based on the actual situation in order to achieve better results.

\section{References}

[1] He F. Study on voltage control and protection of $10 \mathrm{kV}$ distribution network in Yanchi. MS thesis. Chongqing University. Chongqing, China; 2007.

[2] Li TY, Zhao Y, Li N, et al. A new detection method of power quality based on HHT. Proceedings of the Chinese Society for Electrical Engineering, 2005; 25(17):52-56.

[3] Yu DJ, Cheng JS, Yang Y. Hilbert-HuangTransformfor Machinery Fault Diagnosis. Beijing: Science Press; 2006.

[4] Li TY, Zhao Y, Qiao XH, et al. The application of HHT in power system fault signal analysis. Transactions of China ElectroTechnical Society, 2005; 20(6):87-91.

[5] Shao C, Wang J, Fan J. An adaptive EMD endpoint continuation method. Electronic Journal, 2007; 35(10):1944-1947. 